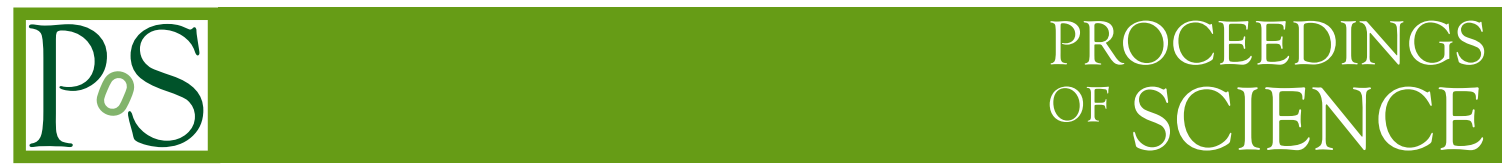

\title{
J-PARC Neutrino Beamline and 1.3 MW Upgrade
}

\author{
Yuichi Oyama*i \\ KEK/J-PARC \\ E-mail: yuichi.oyama@kek.jp
}

J-PARC neutrino beamline has been operated since 2009. Until May 2018, the maximum beam power achieved was $\sim 500 \mathrm{~kW}$. In future, the beam power will be upgraded to $1.3 \mathrm{MW}$. After the stable operation in late 2020 s, we can accumulate $\sim 30 \times 10^{20}$ POT data per year. It is almost the same number as total POT accumulated in recent 10 years. The upgrade plan of the Main Ring as well as the neutrino beamline is reported.

The 21st international workshop on neutrinos from accelerators (NuFact2019)

August 26 - August 31, 2019

Daegu, Korea

\footnotetext{
${ }^{*}$ Speaker.

${ }^{\dagger}$ for T2K neutrino beamline group
} 


\section{Introduction}

J-PARC neutrino beamline has been operated since 2009. Until May 2018, $31.6 \times 10^{20}$ POT (proton of target) of $30 \mathrm{GeV}$ proton beam were supplied to the T2K experiment. The maximum beam power was $\sim 500 \mathrm{~kW}$.

In future, the beam power will be upgraded to $1300 \mathrm{~kW}$. For this purpose, the Main Ring will provide $1300 \mathrm{~kW}$ beam to the neutrino beamline. In addition, the neutrino beamline must accept $1300 \mathrm{~kW}$ beam from the Main Ring. The upgrade plans of the Main Ring as well as the neutrino beamline are reported in this article. Detailed report about the upgrade plan can be found in the Technical Design Report (TDR)[1]. Also some of the upgrade components were precisely reported in other presentations $[2,3,4]$.

\section{Main Ring}

The beam power is inversely proportional to the repetition cycle, and is proportional to number of protons per pulse. At present, the repetition cycle and number of protons per pulse are $2.48 \mathrm{~s}$ and $2.6 \times 10^{14}$, respectively. In future, they will be upgraded to be $1.16 \mathrm{~s}$ and $3.2 \times 10^{14}$.

For the change of the repetition cycle and the number of protons per pulse, two Main Ring upgrades are in progress. These are replacements of power supplies for main magnets and RF cavities. Other changes such as the collimators and the fast extraction kicker are reported in [1], and are not covered in this paper.

\subsection{Power supplies for main magnets}

For the short repetition cycle, fast energy recovery with large capacitor banks is required. New power supplies with large capacitor banks are under preparation. Some of the new power supplies were already installed in the present system and have been used for the regular operation. New power supply buildings, namely, D4, D5 and D6, were already constructed by Japanese Fiscal Year (JFY) 2017. All construction, installation and commissioning will be completed by March 2022. For this purpose, a long shutdown will be needed in 2021.

\subsection{RF cavities}

Higher RF voltages are necessary both for the short repetition cycle, and for large number of protons per pulse. At present, 7 fundamental RF cavities and 2 second harmonic RF cavities are used for $2.48 \mathrm{~s}$ repetition cycle. For $1.16 \mathrm{~s}$ repetition cycle, 11 fundamental RF cavities and 2 new-type second harmonic RF cavities are needed. New-type second harmonic RF cavities were assembled. They will be installed during the long shutdown in 2021. Additional fundamental RF cavities will be installed one by one afterward.

After about one year shutdown in 2021, the beam power will be upgraded to $700 \mathrm{~kW}$ in 2022 . After 2022, additional RF magnets will be installed one by one, and, hopefully, the beam power will be $1300 \mathrm{~kW}$ in 2028 . 


\section{Neutrino beamline}

A detailed description about the J-PARC neutrino beamline at the beginning of the T2K experiment can be found in [5]. Upgrades for some of the components are planned and are in progress. These are in the following subsections.

\subsection{Target}

The target is made of graphite with a cylindrical shape $(26 \mathrm{~mm} \phi \times 900 \mathrm{~mm})$ which is embedded in the first magnetic horn. The present target was designed to accept up to $900 \mathrm{~kW}$ beam.

For higher beam power, the cooling system with helium gas must be changed. The pressure of helium gas should be upgraded from 1.6 bar to $\sim 5$ bar. It improves the helium mass flow rate from $32 \mathrm{~g} / \mathrm{s}$ to $60 \mathrm{~g} / \mathrm{s}$. These estimations were obtained from simulation of the thermal stress and the cooling capacity. The upgraded cooling system with new helium compressors is under design. In the new design, other changes are also considered. Specially, safety and easiness in the remote maintenance is a key issue in the design.

\subsection{Magnetic horns}

In the future operation, larger horn current and higher repetition cycle are required. The horn current will be changed from $250 \mathrm{kA}$ to $320 \mathrm{kA}$, and the repetition cycle must be changed from 2.48 s to $1.16 \mathrm{~s}$ in accordance with the accelerator cycle.

For these purposes, the electrical system of the magnetic horn must be improved. At present, electrical power for the second and the third magnetic horn are supplied from one power supply system. "One horn - one supply" configuration (shown in Figure 1) is needed to satisfy above requirements.

New power supplies, new transformers, and new low impedance striplines are already designed. To extend the cooling capacity for horn conductors, cooling water from striplines is under consideration.

\subsection{Beam window}

The beam window separates helium vessel from vacuum in the primary beamline. It is a double wall of $0.3 \mathrm{~mm}$ thick Ti-6Al-4V, cooled by helium gas $(0.8 \mathrm{~g} / \mathrm{s})$. The most serious problem is that stress by partial heat load at the beam spot may break the window.

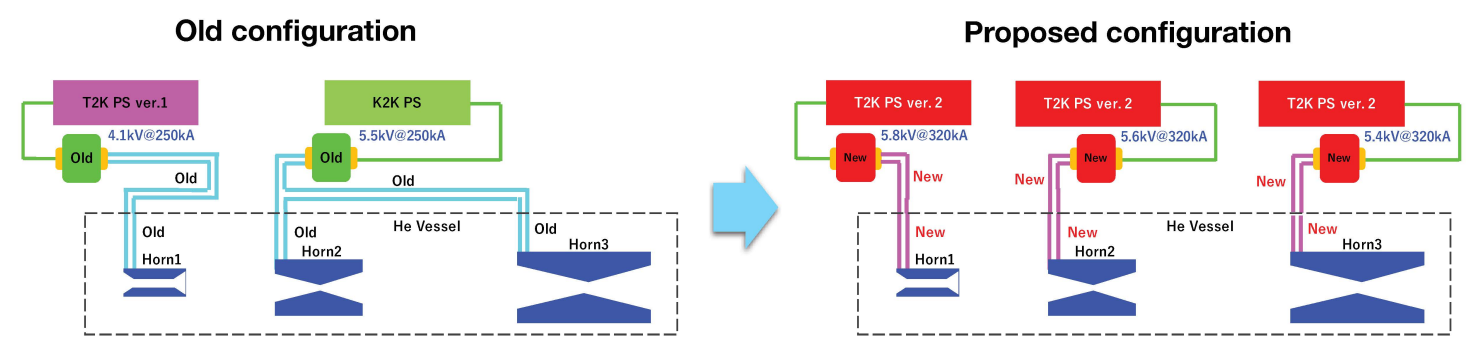

Figure 1: Schematic illustration of electrical system for the magnetic horns. Present configuration (left) and future upgrade (right) are shown. 
The current beam window can accept up to $\sim 750 \mathrm{~kW}$ beam. The thickness of the beam window will be changed to $>0.4 \mathrm{~mm}$ to improve the tolerance for thermal stress in the next version.

Radiation damage may change the characteristics of the thermal stress of the material. It is an open question, and is under study as given in the next subsection.

\subsection{Study of radiation damage}

The radiation damage of the Ti-6Al-4V has been reported only up to $0.3 \sim 0.4$ DPA (displacement per atom). It corresponds to $2 \times 10^{20} \mathrm{POT}$. When the first beam window was replaced in $\mathrm{T} 2 \mathrm{~K}$, $22.4 \times 10^{20}$ POT had been delivered. The radiation damage of $\mathrm{T} 2 \mathrm{~K}$ target/window were already beyond the existing damage data.

RaDIATE International collaboration[6] is studying the effect of radiation damage on target/window materials since 2012. J-PARC officially joined the project in 2017. Irradiation of various material samples at BLIP (BNL) have been studied. Use of other materials for beam window and other beamline components is under investigation based on the experimental data.

\subsection{Cooling water for helium vessel, decay volume and beam dump}

Large fractions of the beam energy are absorbed in the helium vessel, the decay volume and the beam dump. Thermal stress may damage the structure of the helium vessel and the decay volume. Their temperature must be kept below $60{ }^{\circ} \mathrm{C}$. The temperature of the beam dump core must be below $400{ }^{\circ} \mathrm{C}$. They are cooled by cooling water system. At present, the system is adjusted for the $1000 \mathrm{~kW}$ operation. The cooling power can be upgraded by increasing flow rate of the cooling water. Some components in the cooling water circulation system must be exchanged or added. They are circulation pumps, heat exchangers, chillers and cooling towers. Radioactivity of the cooling water will become higher. This is another serious problem.

\subsection{Drainage of radio-active water}

About $20 \mathrm{~m}^{3}$ of cooling water in the neutrino beam line is exposed to neutrons from the proton beam, and are highly activated. Active water are stored in a $\sim 20 \mathrm{~m}^{3}$ of a buffer tank. Then they are sent to two $50 \mathrm{~m}^{3}$ disposal tanks and diluted to satisfy tritium concentration $<42 \mathrm{~Bq} / \mathrm{cc}$, which is required from a regulation for drainage. The drainage can be done every three business day cycle. Present disposal capability is $\sim 180 \mathrm{GBq} /$ year of tritium. However, $\sim 600 \mathrm{GBq} /$ year of tritium are produced for $1300 \mathrm{~kW}$ beam. The present disposal tanks are too small, and we are planning to construct two $\sim 200 \mathrm{~m}^{3}$ disposal tanks.

\section{Summary and outlook}

All upgrades in the neutrino beamline will be finished by Mar. 2022. The beamline will wait for delivery of $1300 \mathrm{~kW}$ beam from the Main Ring. The target, magnetic horns and beam windows need periodical exchange. Additional upgrades will be needed at the exchanges after 2022. After $1300 \mathrm{~kW}$ beam operation become stable, we can accumulate $\sim 30 \times 10^{20}$ POT data per year as shown in Figure 2. It is almost the same number as total POT accumulated in $\sim 10$ years until 2019. 


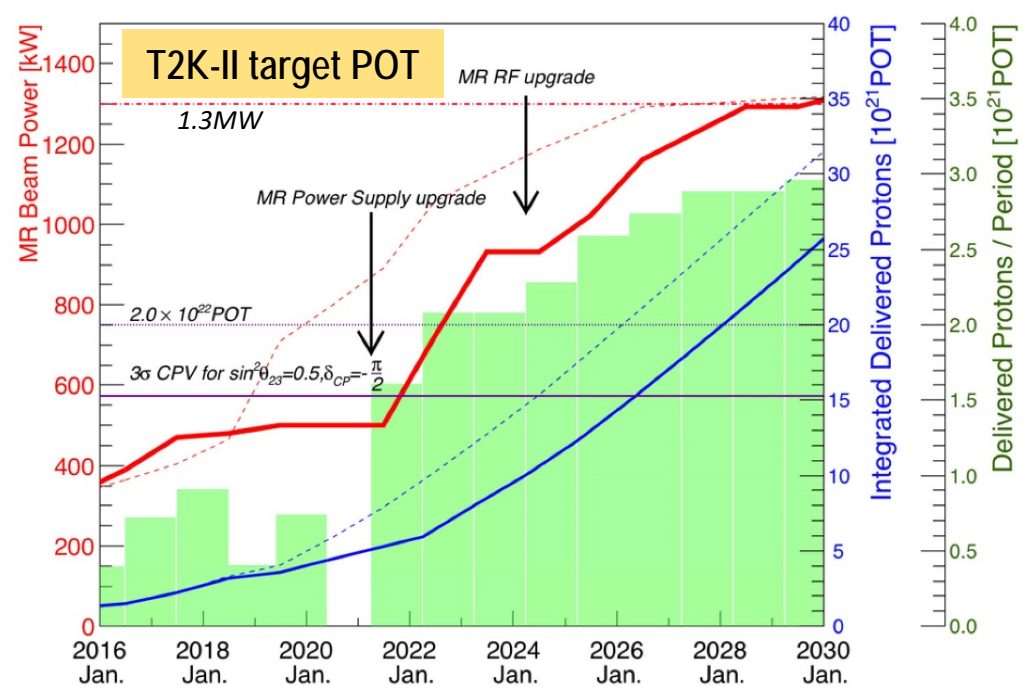

Figure 2: The target MR beam power and accumulated POT as a function of Japanese Fiscal Year (JFY). The solid lines are the target MR beam power (red) and accumulated POT (blue) where six months of the MR operation with the fast-extraction mode each year and the running time efficiency of $90 \%$ are assumed.

\section{References}

[1] K.Abe et al.(The T2K collaboration and the J-PARC neutrino facility group), arXiv:1908.05141[physics.ins-det](2019).

[2] Yoichi Sato, "The current status KEK and J-PARC accelerators for neutrino experiments", in these proceedings.

[3] Megan Friend, "Development of New Proton Beam Monitors for J-PARC 1.3 MW Upgrade", in these proceedings.

[4] Kenji Yasutome, "Development of New Muon Monitors for J-PARC Neutrino Experiment", in these proceedings.

[5] K.Abe et al.(The T2K collaboration), Nucl.Instru.Meth. A 659 106-135(2011).

[6] RaDIATE collaboration, https : //radiate.fnal.gov/ 